\title{
Nanoscale
}

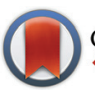

CrossMark \& click for updates

Cite this: Nanoscale, 2015, 7, 18708

\section{Anomalous polarization dependence of Raman scattering and crystallographic orientation of black phosphorus $\uparrow$}

\author{
Jungcheol Kim,,$^{a}$ Jae-Ung Lee, $t^{a}$ Jinhwan Lee, $t^{\mathrm{b}}$ Hyo Ju Park, ${ }^{\mathrm{C}}$ Zonghoon Lee, ${ }^{\mathrm{c}}$ \\ Changgu Lee*b,d and Hyeonsik Cheong*a
}

\begin{abstract}
We investigated polarization dependence of the Raman modes in black phosphorus (BP) using five different excitation wavelengths. The crystallographic orientation was determined by comparing polarized optical microscopy with high-resolution transmission electron microscopy analysis. In polarized Raman spectroscopy, the $B_{2 g}$ mode shows the same polarization dependence regardless of the excitation wavelength or the sample thickness. On the other hand, the $\mathrm{A}_{\mathrm{g}}{ }^{1}$ and $\mathrm{A}_{\mathrm{g}}{ }^{2}$ modes show a peculiar polarization behavior that depends on the excitation wavelength and the sample thickness. The thickness dependence can be explained by considering the anisotropic interference effect due to the birefringence and dichroism of the BP crystal, but the wavelength dependence cannot be explained. We propose a simple and failproof procedure to determine the orientation of a BP crystal by combining polarized Raman scattering with polarized optical microscopy.
\end{abstract}

Received 30th June 2015 Accepted 9th October 2015

DOI: $10.1039 / c 5 n r 04349 b$

www.rsc.org/nanoscale ration procedures. Polarized Raman spectroscopy in combination with uniaxial strain is often used to determine the crystallographic orientation of 2-dimensional materials. ${ }^{9-12}$ Because BP is anisotropic, one may expect that polarized Raman spectroscopy without strain can be used to determine the orientation of BP crystals. However, as we report here, the Raman modes in BP crystals show peculiar polarization behaviors that depend on the excitation wavelength and the sample thickness. Unless these dependences are carefully accounted for, one may make incorrect determination of the orientation. We show that in order to determine the orientation unambiguously, a short-wavelength excitation should be used or the interference effect should be explicitly accounted for. In addition, we propose an easy and fail-proof optical method to determine the crystallographic orientation of BP crystals by combining polarized optical microscopy and polarized Raman spectroscopy.

\footnotetext{
${ }^{a}$ Department of Physics, Sogang University, Seoul 121-742, Korea.

E-mail: hcheong@sogang.ac.kr

${ }^{b}$ Department of Mechanical Engineering and Center for Human Interface Nano Technology (HINT), Sungkyunkwan University, Suwon, 440-746, Korea.

E-mail: peterlee@skku.edu

${ }^{c}$ School of Materials Science and Engineering, Ulsan National Institute of Science and Technology (UNIST), Ulsan 689-798, Korea

${ }^{d}$ SKKU Advanced Institute of Nanotechnology (SAINT), Sungkyunkwan University, Suwon, 440-746, Korea

$\dagger$ Electronic supplementary information (ESI) available. See DOI: 10.1039/ c5nr04349b

$\$$ These authors have contributed equally.
}

\section{Results and discussion}

\section{Electron and optical microscopy}

Among exfoliated BP samples, some flakes with long straight edges are found. In Fig. 1(a-d), such a flake is placed on a TEM grid and observed under a cross-polarized optical microscope in the reflection configuration. The incident light is polarized along the horizontal direction. When the long straight edge is parallel or orthogonal to the incident polarization, the sample appears dark, whereas it appears bright in 

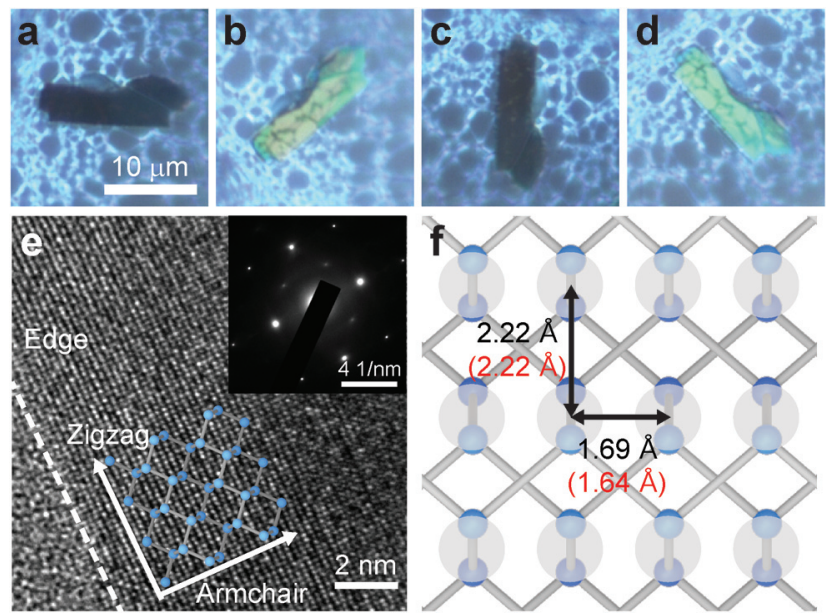

Fig. 1 (a-d) Polarized optical microscopy images of a BP crystal on a TEM grid. Incident light polarization (horizontal in the images) and the analyzer are orthogonal (cross polarization). (e) HR-TEM and TED (inset) images near the long straight edge of the sample. The dashed line is along the long straight edge. (f) Top view of the BP crystal structure. Two stacked layers are shown. Measured (black) and calculated (red) lateral atomic spacings are indicated.

intermediate directions. This effect will be discussed in more detail below. Fig. 1(e) is the high-resolution TEM (HR-TEM) image and the corresponding transmission electron diffraction (TED) pattern, respectively, near the long straight edge. It is established that the long straight edge is along the zigzag direction. This is consistent with a previous calculation that predicted that the ideal strength is much weaker for a tensile strain in the armchair direction, so that it is more likely that a crystal would cleave along the perpendicular direction, i.e., the zigzag direction. ${ }^{13}$ Fig. 1(f) shows the measured lateral atomic spacings which have similar values as the theoretical ones. ${ }^{6}$

The optical contrast of BP crystals was studied in more detail by using cross-polarized optical microscopy on another sample of $\sim 90 \mathrm{~nm}$ thickness. In Fig. 2, the incident light polarization is along the horizontal direction and the analyzer is set along the vertical direction (cross polarization). The sample appears bright when the angle between the incident polarization and the long straight edge is 45 or 135 degrees, whereas it is dark when the angle is 0 or 90 degrees. In parallel polarization, the brightness of the sample does not change much because the surface reflection is rather strong regardless of the polarization direction. The behavior under cross polarization can be explained by birefringence. The crystal structure of bulk $\mathrm{BP}$ is orthorhombic (Cmce), ${ }^{14,15}$ and the refractive indices along the three principal axes are different. ${ }^{16}$ When linearly polarized light goes through a biaxial crystal with the polarization direction not parallel to one of the principal axes, the transmitted light becomes elliptically polarized, and thus optical contrast is observed even under cross polarization. The perpendicular component of the transmitted or reflected light is maximum when the angle between the incident polarization and the principal axis is $45^{\circ}$ or $135^{\circ}$. From the comparison
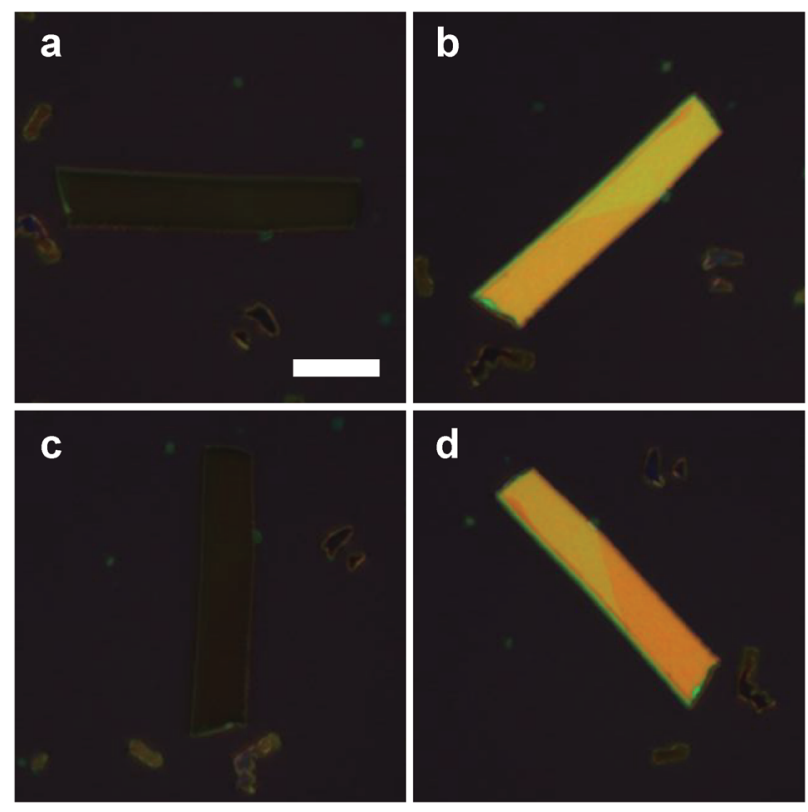

Fig. 2 Cross-polarized optical images of a BP crystal (thickness $\sim 90 \mathrm{~nm}$ ) on a $\mathrm{SiO}_{2} / \mathrm{Si}$ substrate. The scale bar is $10 \mu \mathrm{m}$. The relative angle between the long straight edge (zigzag direction) and the incident polarization direction (horizontal) is (a) $0^{\circ}$, (b) $45^{\circ}$, (c) $90^{\circ}$, and (d) $135^{\circ}$.

between these results with the TEM analysis of Fig. 1, we can determine that the long straight edge in this sample is also along the zigzag direction.

Upon close inspection, we find that the sample appears slightly brighter for $45^{\circ}$ than for $135^{\circ}$ (Fig. S1†). We compared several samples in both transmission and reflection configurations (Fig. S2 and S3 $\dagger$ ) and found that the difference between $45^{\circ}$ and $135^{\circ}$ varies among the samples. In transmission, the contrast difference between $45^{\circ}$ and $135^{\circ}$ is negligible. But in reflection, the contrast difference is often fairly significant (Fig. S2 $\uparrow$ ). This cannot be explained by simple (linear) birefringence because the two directions are symmetric in bulk BP. A possible effect of slight tilting of the sample with respect to the optical axis of the microscope was checked by intentionally tilting a sample. The effect was minimal. Circular dichroism or birefringence would explain the difference, but such chiral properties are not expected in BP due to symmetry. If symmetry is broken due to surface reconstruction, ${ }^{17}$ it may become possible. We observed that the difference increased with time for a given sample, which implies that surface contamination may be responsible for the effect. Further investigation is needed to understand this phenomenon.

\section{Polarized Raman spectroscopy}

Polarized Raman measurements were carried out on the sample in Fig. 2. Fig. 3(a) shows the crystal structure of BP. The zigzag direction is chosen to be along the $x$ axis, and the armchair direction along the $z$ axis. For polarized Raman measurements, the analyzer is set parallel to the incident polarization direction, and the spectra were measured as a 
a
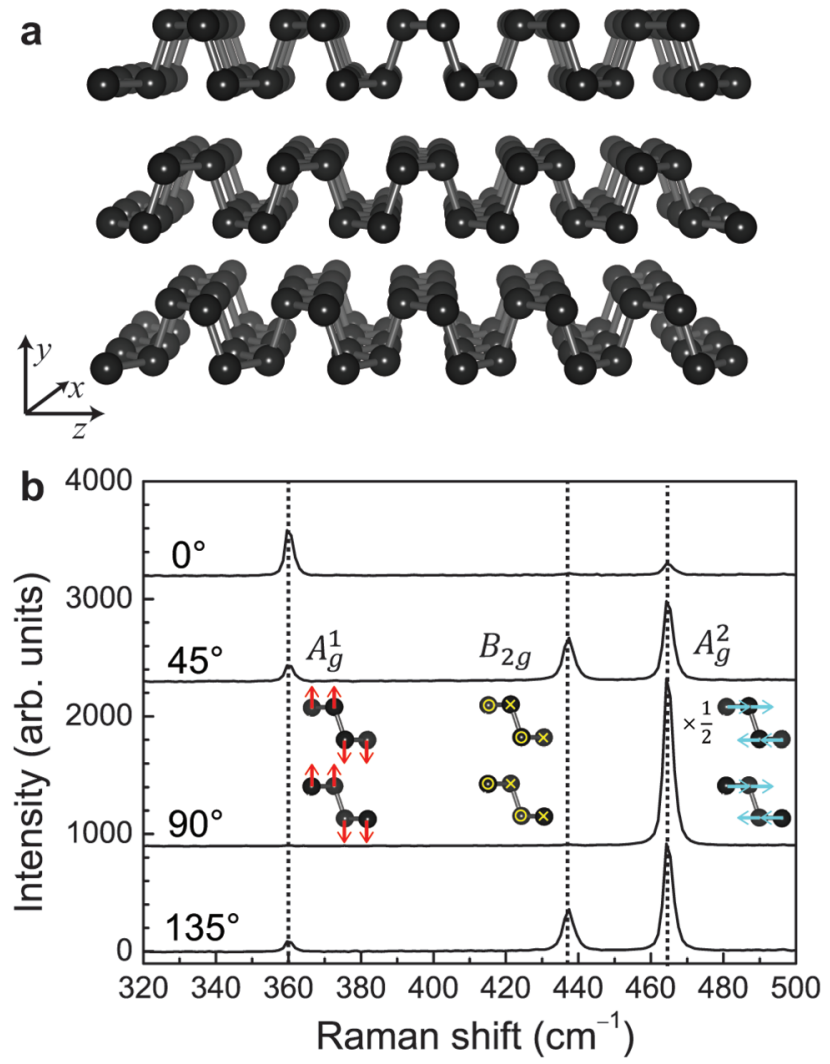

Fig. 3 (a) Crystal structure of BP. The principal axes are shown. (b) Polarized Raman spectra (parallel polarization) of BP measured with $441.6 \mathrm{~nm}$ excitation. The angle between the incident polarization and the zigzag direction for each spectrum is indicated.

function of the incident polarization with respect to the zigzag $(x)$ direction.

Fig. 3(b) shows some representative polarized Raman spectra of BP measured with $441.6 \mathrm{~nm}$ excitation. Three major peaks are observed. The peak at $360 \mathrm{~cm}^{-1}$ is the strongest at $0^{\circ}$ and disappears at $90^{\circ}$. The peak at $437 \mathrm{~cm}^{-1}$, on the other hand, disappears at $0^{\circ}$ and $90^{\circ}$. The strongest peak at $464 \mathrm{~cm}^{-1}$ is maximum at $90^{\circ}$ and minimum at $0^{\circ}$, which is orthogonal to the one at $360 \mathrm{~cm}^{-1}$. The polarization dependence of the peaks can be compared with group theoretical analysis to assign the modes as $\mathrm{A}_{\mathrm{g}}{ }^{1}, \mathrm{~B}_{2 \mathrm{~g}}$, and $\mathrm{A}_{\mathrm{g}}{ }^{2}$, respectively. The vibrational modes are shown schematically in Fig. 3(b).

The intensity of a polarized Raman signal is proportional to, $\left|\hat{e}_{\mathrm{i}} \cdot R \cdot \hat{e}_{\mathrm{s}}\right|^{2}$ where $\hat{e}_{\mathrm{i}}$ and $\hat{e}_{\mathrm{s}}$ are the polarizations of the incident and scattered photons, respectively, and $R$ is the Raman tensor for a given mode. The Raman tensors of the Raman active modes in the backscattering geometry in the complex form are: ${ }^{14,15,18-20}$

$$
\begin{aligned}
R\left(\mathrm{~A}_{\mathrm{g}}\right) & =\left(\begin{array}{ccc}
|a| e^{i \phi_{a}} & 0 & 0 \\
0 & |b| e^{i \phi_{b}} & 0 \\
0 & 0 & |c| e^{i \phi_{c}}
\end{array}\right) \text { and } \\
R\left(\mathrm{~B}_{2 g}\right) & =\left(\begin{array}{ccc}
0 & 0 & |e| e^{i \phi_{e}} \\
0 & 0 & 0 \\
|e| e^{i \phi_{e}} & 0 & 0
\end{array}\right) .
\end{aligned}
$$

It should be noted that the polarization dependence for parallel and cross polarizations cannot be fitted simultaneously without taking complex values for the tensor elements. The polarization vectors are given by $\hat{e}_{\mathrm{i}}=\hat{e}_{\mathrm{s}}=$ $(\cos \theta, 0, \sin \theta)$ in a backscattering geometry with parallel polarizations, where the angle $\theta$ is measured with respect to the zigzag direction. Then the Raman intensity is given by

$$
I\left(\mathrm{~A}_{g}\right) \propto\left(|a| \cos ^{2} \theta+|c| \cos \phi_{c a} \sin ^{2} \theta\right)^{2}+|c|^{2} \sin ^{2} \phi_{c a} \cos ^{4} \theta
$$

and

$$
I\left(\mathrm{~B}_{2 \mathrm{~g}}\right) \propto 4|e|^{2} \cos ^{2} \theta \sin ^{2} \theta,
$$

where $\phi_{c a}=\phi_{c}-\phi_{a}$. For cross polarization, $\hat{e}_{\mathrm{i}}=(\cos \theta, 0, \sin \theta)$ and $\hat{e}_{\mathrm{s}}=\left(\cos \left(\theta+90^{\circ}\right), 0, \sin \left(\theta+90^{\circ}\right)\right)=(-\sin \theta, 0, \cos \theta)$. The Raman intensity is given by

$$
I\left(\mathrm{~A}_{g}\right) \propto\left(|a|-|c| \cos \phi_{c a}\right)^{2}+|c|^{2} \sin \phi_{c a} \cos ^{2} \theta
$$

and

$$
I\left(\mathrm{~B}_{2 g}\right) \propto|e|^{2}\left(\cos ^{2} \theta-\sin ^{2} \theta\right)^{2} .
$$

It is obvious that the zigzag $\left(0^{\circ}\right)$ and the armchair $\left(90^{\circ}\right)$ directions are clearly distinguished in the above analysis and the data in Fig. 3(b). These results are similar to previous reports. ${ }^{2,19,21,22}$ However, as we shall see in the following, the polarization dependence of the modes is strongly influenced by the excitation wavelength and the thickness of the sample, and the crystallographic orientations cannot be determined unambiguously without taking this fact into account.

We repeated the polarized Raman measurements with different excitation lasers. Fig. 4 summarizes the polarization dependence of the $\mathrm{A}_{\mathrm{g}}{ }^{1}, \mathrm{~A}_{\mathrm{g}}{ }^{2}$, and $\mathrm{B}_{2 \mathrm{~g}}$ modes for excitation wavelengths of 441.6, 488, 514.5, 532, and $632.8 \mathrm{~nm}$ in parallel polarization. Similar data for cross polarization are shown in Fig. S4. $\dagger$ The angles are measured with respect to the zigzag $(x)$ direction. It is striking that the polarization dependence of the $\mathrm{A}_{\mathrm{g}}$ modes dramatically changes with the excitation wavelength. The $\mathrm{A}_{\mathrm{g}}{ }^{1}$ mode, for example, shows a bow-tie shape dependence with the maximum at $0^{\circ}$ for $441.6 \mathrm{~nm}$. However, the same mode is almost isotropic for 514.5 or $532 \mathrm{~nm}$. The $\mathrm{A}_{\mathrm{g}}{ }^{2}$ mode is even more striking: the maximum occurs at $90^{\circ}$ for all the excitation wavelengths except for $632.8 \mathrm{~nm}$ for which the maximum is at $0^{\circ}$. The $\mathrm{B}_{2 g}$ mode, on the other hand, has the same polarization dependence regardless of the excitation wavelength. We repeated the measurements with several samples with different thicknesses using $441.6 \mathrm{~nm}$ and $514.5 \mathrm{~nm}$ excitation wavelengths. The results are summarized in Fig. 5 and the detailed spectra are shown in Fig. S5. $\dagger$ For different thicknesses, the polarization dependence for each mode is fairly similar for $441.6 \mathrm{~nm}$ excitation, whereas it varies greatly with thickness for $514.5 \mathrm{~nm}$ excitation.

The effect of optical interference ${ }^{23}$ may explain this excitation wavelength and thickness dependence as both the wavelength and the sample thickness are the determining factors 

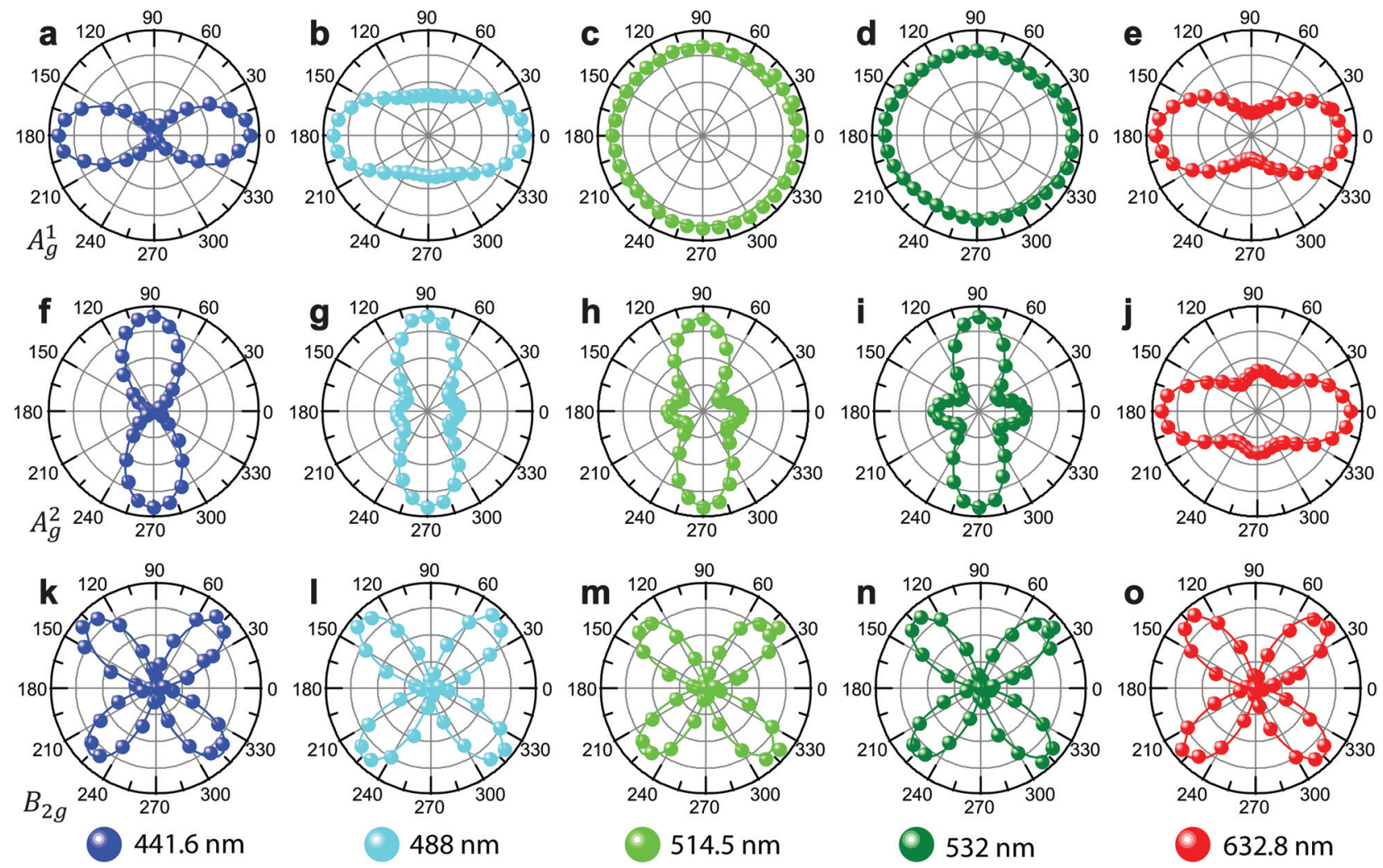

Fig. 4 Polarization dependence of Raman modes for different excitation wavelengths. Each row shows polarization dependence of $A_{g}{ }^{1}$, $A_{g}{ }^{2}$ and $B_{2 g}$ modes, respectively, taken with excitation wavelengths of $441.6,488,514.5,532$, and $632.8 \mathrm{~nm}$ as indicated. The curves represent best fits to the calculated polarization dependence of the Raman intensities using eqn. (2) and (3). The polarization behaviors of the $A_{g}$ modes exhibit strong dependence on the excitation wavelength, whereas the $B_{2 g}$ mode does not show such dependence.

of interference. Because BP is an anisotropic material, it has both linear dichroism and birefringence, both of which contribute to polarization dependent interference. By using the reported refractive indices ${ }^{24}$ we calculate the interference enhancement factors of the $A_{g}$ modes along the zigzag and armchair directions. $\dagger$ As shown in Fig. 6(a), the enhancement factors vary greatly with thickness and are dramatically different along the two principal axes. Fig. 6(b) shows the ratio of the enhancement factors along the zigzag and the armchair directions. When the incident polarization is along the zigzag direction, the Raman intensity can be enhanced much more than in the armchair polarization case. Note that for $441.6 \mathrm{~nm}$ excitation, the enhancement ratio does not vary much with thickness. This is because of the large imaginary part of the refractive index, $\kappa$, of BP at this wavelength. A large value of $\kappa$ suppresses multiple orders of interference and hence the interference enhancement. Since $\kappa$ increases steeply between $488 \mathrm{~nm}$ and $441.6 \mathrm{~nm}$ (see Table S1†), ${ }^{24}$ the effect of interference is strong for $488 \mathrm{~nm}$ and suppressed for $441.6 \mathrm{~nm}$. Now one can plot the polarization dependence of the Raman modes with the interference effect corrected for. Fig. 6(c-j) compare the effect of such corrections. After correction, the polarization dependence measured with $514.5 \mathrm{~nm}$ excitation becomes fairly similar for the samples with different thick- nesses [Fig. 6(d and f)]. Therefore, the sample thickness dependence can be explained as being due to anisotropic interference. However, for the different excitation wavelengths, the $\mathrm{A}_{\mathrm{g}}{ }^{1}$ and $\mathrm{A}_{\mathrm{g}}{ }^{2}$ modes still show qualitatively different polarization behaviors even after the correction for interference. Because of the anisotropy in the band structure, it is reasonable to expect that the Raman tensor elements vary with the excitation wavelength. In isotropic materials, the resonance effect reflects such an effect. In an anisotropic material such as BP, the excitation energy dependence of the tensor elements would result in variations in the polarization behaviors, and the $|a| /|c|$ ratio would change with the excitation wavelength. Such an effect is not observed in isotropic 2-dimensional materials such as graphene or $\operatorname{MoS}_{2}{ }^{25,26}$ On the other hand, because $R\left(\mathrm{~B}_{2 g}\right)$ has only one element $e$, the functional form of the polarization dependence of the $B_{2 g}$ mode will not change even if the magnitude of $e$ changes. This would explain the observed difference between the $\mathrm{A}_{\mathrm{g}}$ modes and the $\mathrm{B}_{2 \mathrm{~g}}$ mode. The polarization dependence for the $\mathrm{A}_{\mathrm{g}}$ modes for each excitation wavelength in Fig. 6(h and j) is fitted to the calculated Raman intensity using eqn. (2) and (3), and the obtained $|a| /|c|$ ratios and $\phi_{c a}$ values are summarized in Table 1.

In order to visualize the thickness dependence, we performed polarized wide-field Raman imaging measurements ${ }^{27}$ 

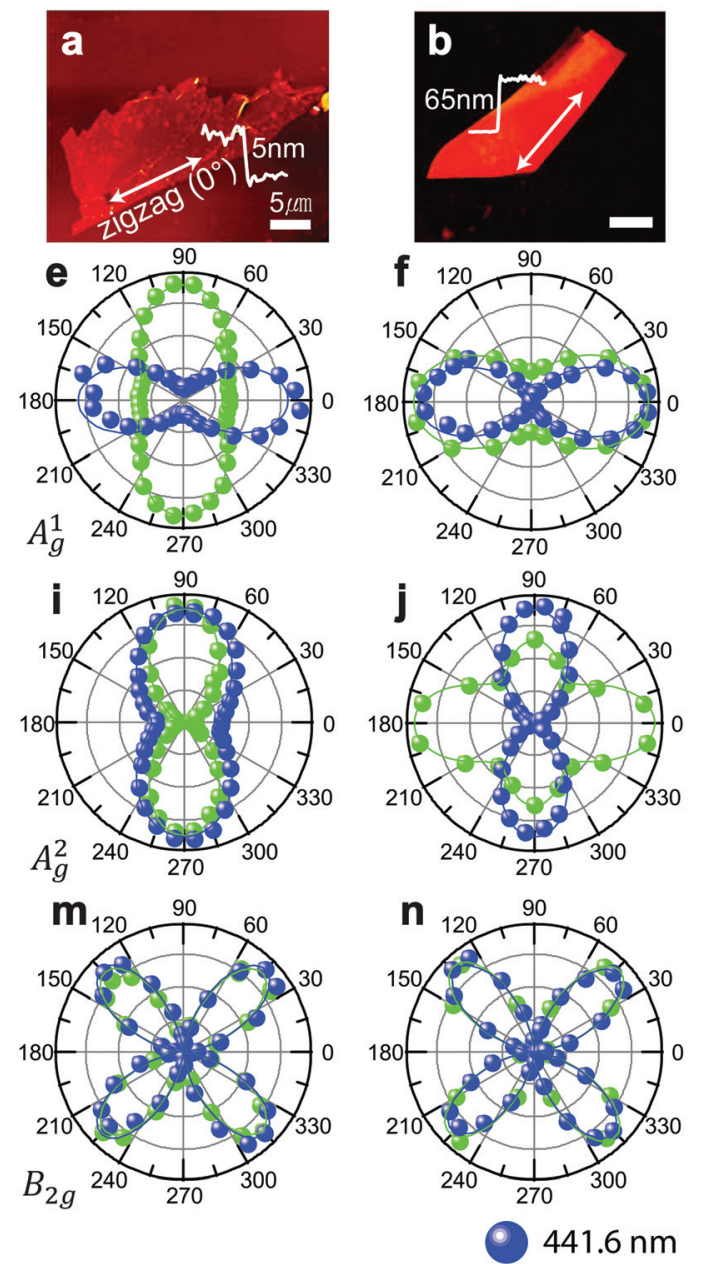
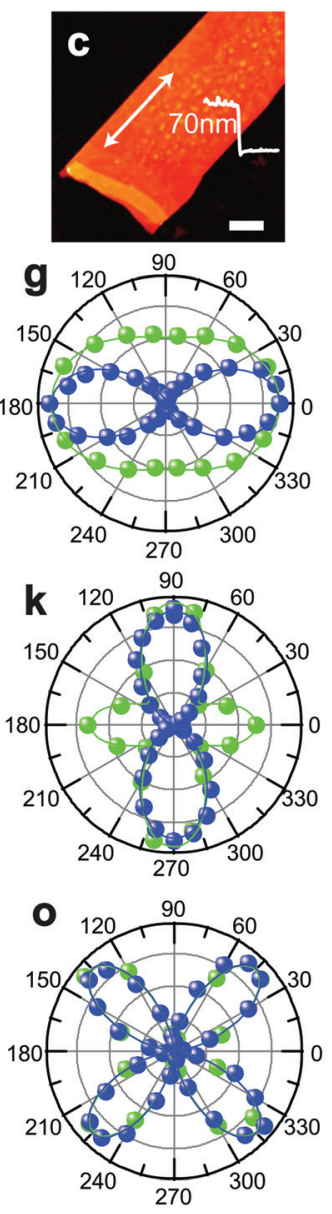

$514.5 \mathrm{~nm}$
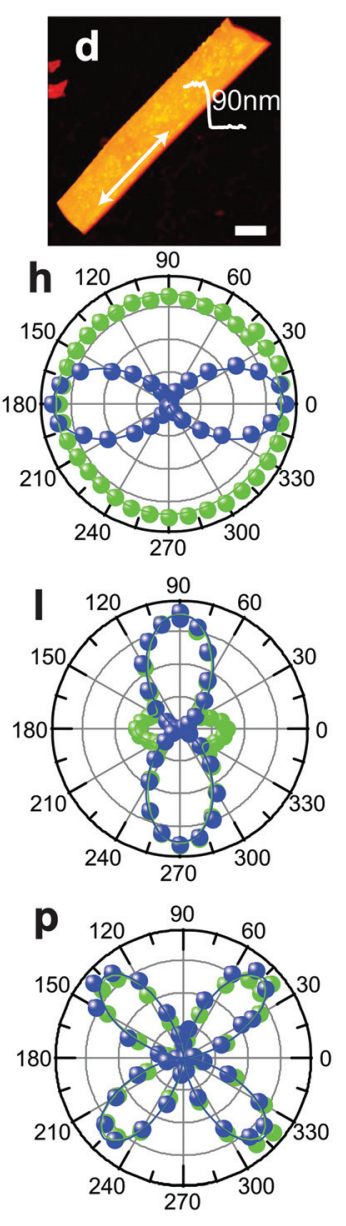

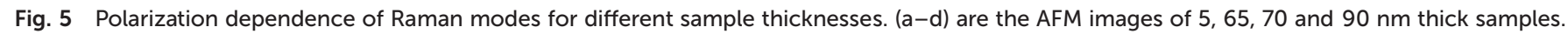
The $5 \mathrm{~nm}$ sample is on a substrate with $100 \mathrm{~nm} \mathrm{SiO}$, and the others are on $300 \mathrm{~nm} \mathrm{SiO}$. Each row shows polarization dependence of $\mathrm{A}_{\mathrm{g}}{ }^{1}$, $\mathrm{A}_{\mathrm{g}}{ }^{2}$ and $B_{2 g}$ modes, respectively, taken with excitation wavelengths of 441.6 and $514.5 \mathrm{~nm}$ as indicated. The curves represent best fits to the calculated polarization dependence of the Raman intensities using eqn (2) and (3). The polarization behaviors of the $A_{g}$ modes exhibit strong dependence on the thickness for $514.5 \mathrm{~nm}$ excitation whereas no such dependence is seen for $441.6 \mathrm{~nm}$ excitation.

on a sample with several BP flakes with different thicknesses using $514.5 \mathrm{~nm}$ excitation. The wide-field Raman images for each mode as the polarization (indicated by the arrow) of the incident laser is rotated are shown in Movie S1. $\dagger$ Fig. 7(a) is an optical image of the sample. BP flakes with different thicknesses appear in different colors. Fig. 7(b) represents the intensity ratio of the $\mathrm{Ag}_{\mathrm{g}}{ }^{2}$ mode intensity taken with the excitation polarization in the horizontal direction to that in the vertical direction. For this measurement, two wide-field Raman images were taken from the same sample area successively in the two polarization directions, and then the ratio image was obtained by processing the images. Fig. 7(b) clearly shows that even in the same flake, areas with different thicknesses have different ratios. Therefore, relying on the polarization behavior of the $A_{g}$ modes alone may lead to incorrect determination of the crystallographic orientation if the excitation wavelength is $514.5 \mathrm{~nm}$ or longer.

\section{Conclusions}

Based on our results, we propose the following procedure to determine the crystallographic orientation of BP flakes. First, the sample should be inspected by using an optical microscope in cross polarization. By rotating the sample until the sample appears dark, one can determine that the zigzag direction $(x)$ is either parallel or perpendicular to the incident polarization or the analyzer direction. At this point, the principal axes of the crystal ( $x$ or $z$ ) are found, but one cannot know which of the two directions the zigzag direction is. Then, two Raman spectra in parallel polarization need to be measured, with the excitation polarization along each of the principal axes. One should use a short wavelength laser (441.6 nm in our case). The direction in which the $\mathrm{Ag}_{\mathrm{g}}{ }^{1}\left(\mathrm{~A}_{\mathrm{g}}{ }^{2}\right)$ mode is stronger (weaker) is the zigzag direction. One can indeed skip the first step with an optical microscope if a full polar plot of one of 

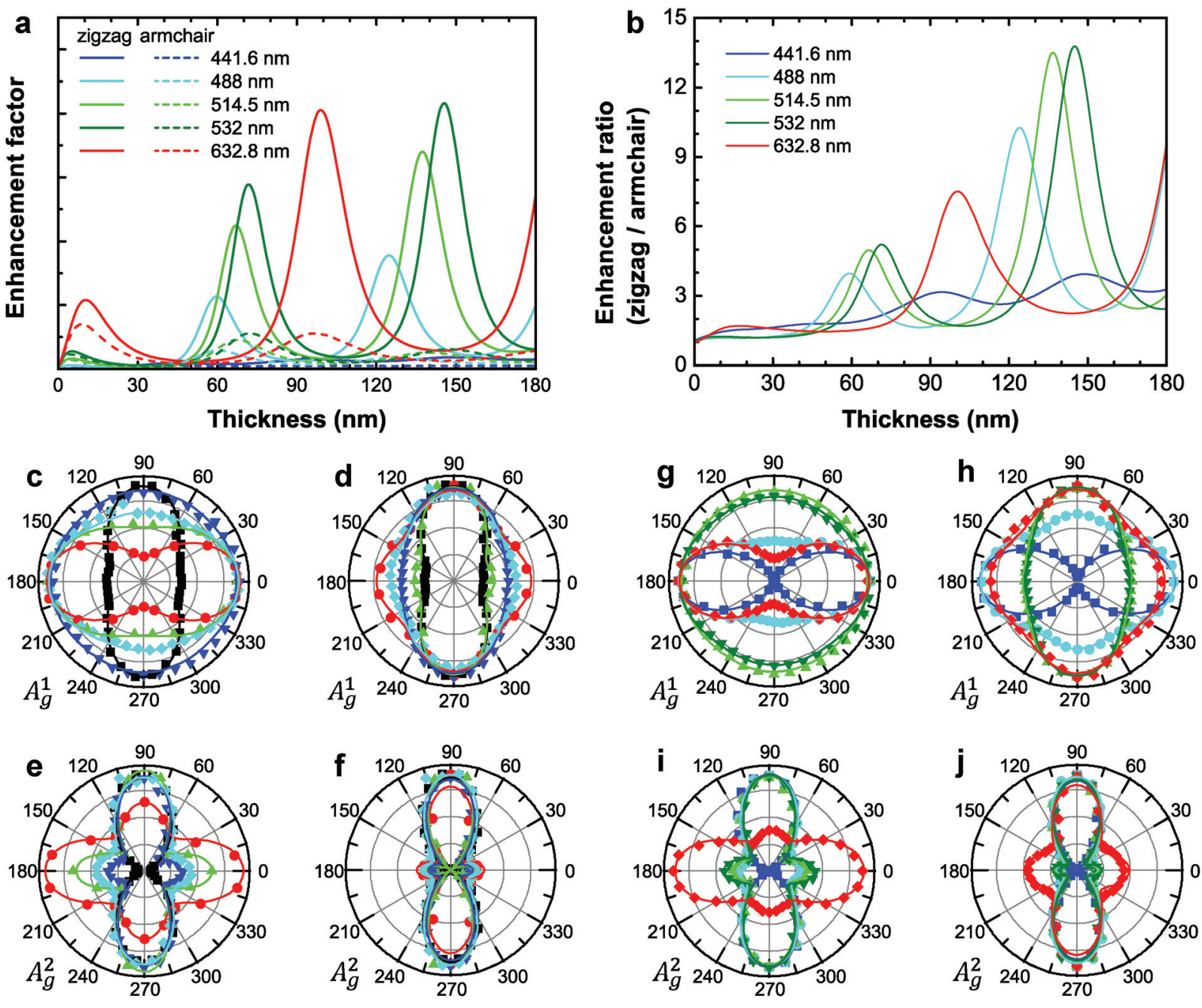

$\square 5 \mathrm{~nm} \bigcirc 65 \mathrm{~nm} \triangle 70 \mathrm{~nm} \nabla 90 \mathrm{~nm} \vee 90 \mathrm{~nm}$ (quartz)

$\square 41.6 \mathrm{~nm} \odot 448 \mathrm{~nm} \triangle 514.5 \mathrm{~nm} \nabla 532 \mathrm{~nm} \diamond 632.8 \mathrm{~nm}$

Fig. 6 (a) Calculated interference enhancement factor as a function of the sample thickness for zigzag $(x)$ and armchair $(z)$ directions for different excitation wavelengths for $\mathrm{BP}$ on $300 \mathrm{~nm} \mathrm{SiO}{ }_{2}$ on the Si substrate. (b) Enhancement ratio obtained by dividing the enhancement factor for the zigzag direction with that for the armchair direction. (c-f) Polarization dependence of $A_{g}$ modes for different thicknesses measured with $514.5 \mathrm{~nm}$ excitation, (c, e) before and (d, f) after the correction for interference. The data include a $5 \mathrm{~nm}$ thick sample on $100 \mathrm{~nm} \mathrm{SiO} 2$ on $\mathrm{Si}$ and a $90 \mathrm{~nm}$ thick sample on quartz. ( $g-j$ ) Polarization dependence of $\mathrm{A}_{\mathrm{g}}$ modes for different excitation wavelengths from a $90 \mathrm{~nm}$ thick sample on $300 \mathrm{~nm} \mathrm{SiO}_{2}$ on $\mathrm{Si}_{\text {, }}$ $(g, i)$ before and $(h, j)$ after the correction for interference.

Table 1 Excitation wavelength dependence of a/c ratios. Ratios of the Raman tensor components $a$ and $c$ were obtained from fitting the data in Fig. 4 to eqn (2) and (3)

\begin{tabular}{|c|c|c|c|c|}
\hline \multirow[b]{2}{*}{ Wavelength (nm) } & \multicolumn{2}{|l|}{$\mathrm{A}_{\mathrm{g}}{ }^{1}$} & \multicolumn{2}{|l|}{$\mathrm{A}_{\mathrm{g}}{ }^{2}$} \\
\hline & $a / c$ & Phase $\left(^{\circ}\right)$ & $a / c$ & Phase $\left(^{\circ}\right)$ \\
\hline 441.6 & 4.01 & 0 & 0.24 & 179 \\
\hline 488 & 1.20 & 48 & 0.47 & 97 \\
\hline 514.5 & 0.76 & 34 & 0.50 & 111 \\
\hline 532 & 0.75 & 43 & 0.52 & 120 \\
\hline 632.8 & 0.98 & 47 & 0.79 & 91 \\
\hline
\end{tabular}

the $\mathrm{A}_{\mathrm{g}}$ modes is measured with a short wavelength laser. However, because many more Raman spectra should be taken, it would be much more time consuming.

\section{Experimental methods}

\section{Sample preparation}

The samples were prepared directly on $\mathrm{SiO}_{2} / \mathrm{Si}$ substrates by mechanical exfoliation from BP flakes (Smart Elements). For HR-TEM measurements, exfoliated BP flakes were transferred from the $\mathrm{Si} / \mathrm{SiO}_{2}$ wafer onto a TEM grid using the wet transfer method. ${ }^{28} \mathrm{~A}$ wafer with BP flakes was coated with poly(methyl methacrylate) (PMMA) at 4000 RPM and separated in a buffered oxide etchant (BOE) solution by etching the $\mathrm{SiO}_{2}$ layer. To prevent contamination, it was washed in DI water several times. Then, BP with the PMMA support was transferred onto a TEM grid (Lacey Formvar/Carbon Coated Copper Grid, SPI Supplies). To remove the PMMA support, the grid sample was put into acetone for $15 \mathrm{~min}$ 

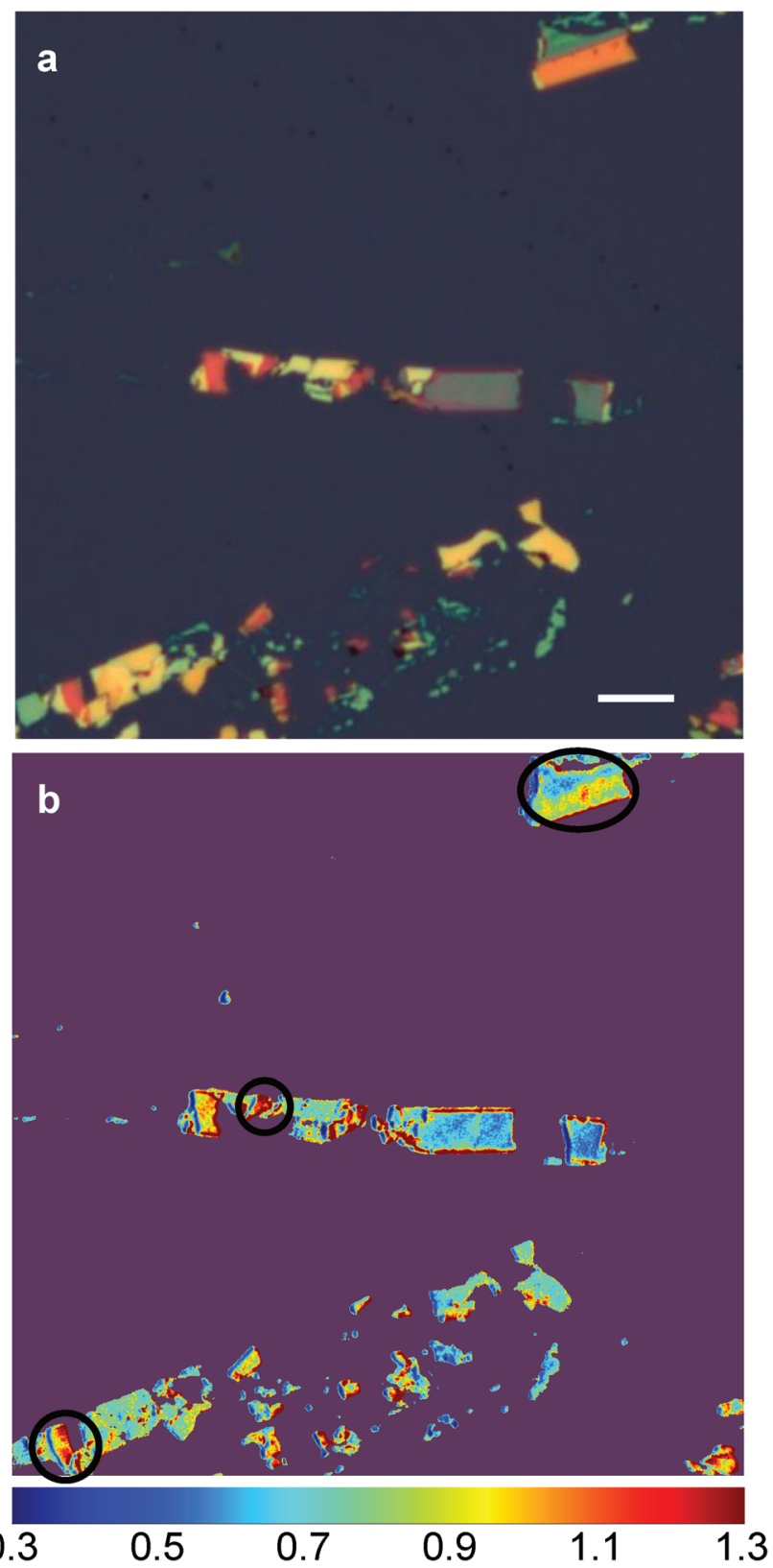

Fig. 7 (a) Optical image of a sample with several BP flakes with different thicknesses. The scale bar is $20 \mu \mathrm{m}$. (b) Polarized Raman intensity ratio image. The Raman intensity of the $\mathrm{Ag}_{\mathrm{g}}{ }^{2}$ mode taken with the excitation polarization in the horizontal direction is divided by that in the vertical direction. The intensity ratio varies greatly even inside a given flake, depending on the thickness (circled areas).

and annealed in a flow of Ar gas at $250{ }^{\circ} \mathrm{C}$ for $9 \mathrm{~h}$. The optical measurements were carried out with BP samples on $\mathrm{SiO}_{2} / \mathrm{Si}$ substrates. The thickness was determined by using an atomic force microscope (NT-MDT NTEGRA Spectra). The samples were kept in an optical vacuum chamber to keep them away from contamination in the atmosphere. ${ }^{29,30}$

\section{Polarized optical microscopy}

A commercial optical microscope (Nikon LV100) was used to obtain the optical images. The incident polarization direction was fixed and the sample was rotated by using a rotation stage. The analyzer was fixed to cross polarization configuration (perpendicular to incident polarization).

\section{HR-TEM measurements}

HR-TEM analysis was carried out using a Cs-corrected highresolution transmission electron microscope (FEI Titan) operated in the range of 80 to $200 \mathrm{kV}$.

\section{Polarized Raman measurements}

We used 5 different excitation sources: the $441.6 \mathrm{~nm}(2.81 \mathrm{eV})$ line of a He-Cd laser, the 488 and $514.5 \mathrm{~nm}$ (2.54 and $2.41 \mathrm{eV}$ ) lines of an Ar ion laser, the $532 \mathrm{~nm}(2.33 \mathrm{eV})$ line of a diode-pumped-solid-state laser, and the $632.8 \mathrm{~nm}(1.96 \mathrm{eV})$ line of a He-Ne laser. To prevent degradation of the sample, ${ }^{29}$ all the measurements were performed while the sample was kept in an optical vacuum chamber (Oxford Microstat He2). The laser beam was focused onto the sample by using a 40× long-working distance microscope objective lens (0.6 N.A.), and the scattered light was collected and collimated by using the same objective. The Raman signal was dispersed with a Jobin-Yvon Horiba TRIAX 550 spectrometer (1800 grooves per $\mathrm{mm}$ ) and detected with a liquid-nitrogencooled back-illuminated charge-coupled-device (CCD) detector. The laser power was kept below $0.5 \mathrm{~mW}$. An achromatic half-wave plate was used to rotate the polarization of the linearly polarized laser beam to the desired direction. For parallel (perpendicular) polarization, the analyzer angle was set such that photons with polarization parallel (perpendicular) to the incident polarization pass through. Another achromatic halfwave plate was placed in front of the spectrometer to keep the polarization direction of the signal entering the spectrometer constant with respect to the groove direction of the grating. ${ }^{25,31}$

To obtain polarized wide field Raman images, a liquidcrystal tunable bandpass filter (Varyspec VISR) and an electron multiplying charge-coupled-device (EMCCD, Andor iXon3 888) were used. For illumination, the incident laser beam was passed through a beam shaper (piShaper 6_6) to make the power density uniform across the illuminated area of $\sim 200 \mu \mathrm{m}$ diameter. The total laser power was $100 \mathrm{~mW}$, and the spectral resolution was $\sim 10 \mathrm{~cm}^{-1}$.

\section{Calculation of enhancement factors by interference effects}

We used the model that Yoon et $a .^{23}$ used to calculate the intensity enhancement of Raman scattered light by the interference effect. The laser beam is absorbed during the multiple reflections. The net absorption term at position $x\left[F_{a b}(x)\right]$ can be expressed as

$F_{a b}(x)=t_{01} \frac{\left(1+r_{12} r_{23} \mathrm{e}^{-2 i \beta_{2}{ }^{\mathrm{ex}}}\right) \mathrm{e}^{-i \beta_{x}{ }^{\mathrm{ex}}}+\left(r_{12}+r_{23} \mathrm{e}^{-i \beta_{2}^{\mathrm{ex}}}\right) \mathrm{e}^{-i\left(2 \beta_{1}^{\text {ex }}-\beta_{x}^{\mathrm{ex}}\right)}}{1+r_{12} r_{23} \mathrm{e}^{-2 i \beta_{2}{ }^{\mathrm{ex}}}+\left(r_{12}+r_{23} \mathrm{e}^{-2 i \beta_{2}^{\mathrm{ex}}}\right) r_{1} \mathrm{e}^{-2 i \beta_{1}{ }^{\mathrm{ex}}}}$, 
where $t_{i j}=2 n_{i} /\left(\tilde{n}_{i}+\tilde{n}_{j}\right)$ and $r_{i j}=\left(\tilde{n}_{i}-\tilde{n}_{j}\right) /\left(\tilde{n}_{i}+\tilde{n}_{j}\right)$ are the Fresnel coefficients at the interfaces of the $i$-th and the $j$-th layer; the indices are assigned as air (0), BP (1), $\mathrm{SiO}_{2}(2)$, and $\mathrm{Si}(3) . \tilde{n}_{i}$ is the complex refractive index for the $i$-th layer. The phase terms $\beta_{x}{ }^{\text {ex }}=2 \pi x \tilde{n}_{1} / \lambda_{\text {ex }}$ and $\beta_{i}{ }^{\text {ex }}=2 \pi d_{i} \tilde{n}_{i} / \lambda_{\text {ex }}$ are included, where $d_{i}$ is the thickness of the $i$-th layer and $\lambda_{\mathrm{ex}}$ is the excitation wavelength.

The net scattering term $\left[F_{\mathrm{sc}}(x)\right]$ by multiple reflections of the Raman signal generated at position $x$ can be written as

$F_{\mathrm{sc}}(x)=t_{10} \frac{\left(1+r_{12} r_{23} \mathrm{e}^{-2 i \beta_{2}^{\mathrm{sc}}}\right) \mathrm{e}^{-i \beta_{x}^{\mathrm{sc}}}+\left(r_{12}+r_{23} \mathrm{e}^{-i \beta_{2}^{\mathrm{sc}}}\right) \mathrm{e}^{-i\left(2 \beta_{1}^{\mathrm{sc}}-\beta_{x}^{\mathrm{sc}}\right)}}{1+r_{12} r_{23} \mathrm{e}^{-2 i \beta_{2}^{\mathrm{sc}}}+\left(r_{12}+r_{23} \mathrm{e}^{-2 i \beta_{2}^{\mathrm{sc}}}\right) r_{1} \mathrm{e}^{-2 i \beta_{1}^{\mathrm{sc}}}}$

where $\beta_{x}^{\mathrm{sc}}=2 \pi x \tilde{n}_{1} / \lambda_{\mathrm{sc}}$ and $\beta_{i}^{\mathrm{sc}}=2 \pi d_{i} \tilde{n}_{1} / \lambda_{\mathrm{sc}}$ are the phase terms with $\lambda_{\mathrm{sc}}$ being the wavelength of the Raman signal. By considering the above two terms, the total enhancement factor $(F)$ is given by

$$
F=N \int_{0}^{d_{1}}\left|F_{a b}(x) F_{\mathrm{sc}}(x)\right|^{2} \mathrm{~d} x
$$

where $N$ is the normalization factor. We used the reported refractive indices by Asahina et $a .^{24}$ Since the refractive indices are different for zigzag and armchair directions of black phosphorus, the enhancement factors are calculated separately. The used values are summarized in Table S1.†

\section{Acknowledgements}

This work was supported by the National Research Foundation (NRF) grants funded by the Korean government (MSIP) (No. 2011-0013461, 2011-0017605, and 2015R1A2A2A01006992) and by a grant (No. 2011-0031630) from the Center for Advanced Soft Electronics under the Global Frontier Research Program of MSIP.

\section{Notes and references}

1 L. Li, Y. Yu, G. J. Ye, Q. Ge, X. Ou, H. Wu, D. Feng, X. H. Chen and Y. Zhang, Nat. Nanotechnol., 2014, 9, 372377.

2 F. Xia, H. Wang and Y. Jia, Nat. Commun., 2014, 5, 4458.

3 H. Liu, A. T. Neal, Z. Zhu, Z. Luo, X. Xu, D. Tománek and P. D. Ye, ACS Nano, 2014, 8, 4033-4041.

4 S. P. Koenig, R. A. Doganov, H. Schmidt, A. H. Castro Neto and B. Özyilmaz, Appl. Phys. Lett., 2014, 104, 103106.

5 J. Na, Y. T. Lee, J. A. Lim, D. K. Hwang, G. Kim, W. K. Choi and Y. Song, ACS Nano, 2014, 8, 11753-11762.

6 J. Qiao, X. Kong, Z.-X. Hu, F. Yang and W. Ji, Nat. Commun., 2014, 5, 4475.

7 T. Low, a. S. Rodin, a. Carvalho, Y. Jiang, H. Wang, F. Xia and a. H. Castro Neto, Phys. Rev. B: Condens. Matter, 2014, 90, 075434.

8 H. Yuan, X. Liu, F. Afshinmanesh and W. Li, Nat. Nanotechnol., 2015, 10, 707-713.

9 T. M. G. Mohiuddin, a. Lombardo, R. R. Nair, a. Bonetti, G. Savini, R. Jalil, N. Bonini, D. M. Basko, C. Galiotis,
N. Marzari, K. S. Novoselov, a. K. Geim and a. C. Ferrari, Phys. Rev. B: Condens. Matter, 2009, 79, 1-8.

10 D. Yoon, Y.-W. Son and H. Cheong, Phys. Rev. Lett., 2011, 106, 155502.

11 M. Huang, H. Yan, C. Chen, D. Song, T. F. Heinz and J. Hone, Proc. Natl. Acad. Sci. U. S. A., 2009, 106, 7304-7308.

12 Y. Wang, C. Cong, C. Qiu and T. Yu, Small, 2013, 9, 28572861.

13 Q. Wei and X. Peng, Appl. Phys. Lett., 2014, 104, 251915.

14 S. Sugai and I. Shirotani, Solid State Commun., 1985, 53, 753-755.

15 J. Ribeiro-Soares, R. M. Almeida, L. G. Cançado, M. S. Dresselhaus and A. Jorio, Phys. Rev. B: Condens. Matter, 2015, 91, 205421.

16 T. Nagahama and M. Kobayashi, J. Phys. Soc. Jpn., 1985, 54, 2096-2099.

17 L. Liang, J. Wang, W. Lin, B. G. Sumpter, V. Meunier and M. Pan, Nano Lett., 2014, 14, 6400-6406.

18 M. I. Aroyo, J. M. Perez-Mato, C. Capillas, E. Kroumova, S. Ivantchev, G. Madariaga, A. Kirov and H. Wondratschek, Z. Kristallogr., 2006, 221, 15-27.

19 H. B. Ribeiro, M. A. Pimenta, C. J. S. De Matos and R. L. Moreira, ACS Nano, 2015, 9, 4270-4276.

20 T. Strach, J. Brunen, B. Lederle, J. Zegenhagen and M. Cardona, Phys. Rev. B: Condens. Matter, 1998, 57, 12921297.

21 S. Zhang, J. Yang, R. Xu, F. Wang, W. Li, M. Ghufran, Y.-W. Zhang, Z. Yu, G. Zhang, Q. Qin and Y. Lu, ACS Nano, 2014, 8, 9590-9596.

22 J. Wu, N. Mao, L. Xie, H. Xu and J. Zhang, Angew. Chem., Int. Ed., 2015, 54, 2366-2369.

23 D. Yoon, H. Moon, Y. W. Son, J. S. Choi, B. H. Park, Y. H. Cha, Y. D. Kim and H. Cheong, Phys. Rev. B: Condens. Matter, 2009, 80, 125422.

24 H. Asahina and a. Morita, J. Phys. C: Solid State Phys., 2000, 17, 1839-1852.

25 J. Lee, N. M. Seck, D. Yoon, S.-M. Choi, Y. Son and H. Cheong, Carbon, 2014, 72, 257-263.

26 Y. Zhao, X. Luo, H. Li, J. Zhang, P. T. Araujo, C. K. Gan, J. Wu, H. Zhang, S. Y. Quek, M. S. Dresselhaus and Q. Xiong, Nano Lett., 2013, 13, 1007-1015.

27 R. W. Havener, S. Y. Ju, L. Brown, Z. Wang, M. Wojcik, C. S. Ruiz-Vargas and J. Park, ACS Nano, 2012, 6, 373-380.

28 W. Regan, N. Alem, B. Alemán, B. Geng, Ç. Girit, L. Maserati, F. Wang, M. Crommie and a. Zettl, Appl. Phys. Lett. , 2010, 96, 2-5.

29 A. Castellanos-Gomez, L. Vicarelli, E. Prada, J. O. Island, K. L. Narasimha-Acharya, S. I. Blanter, D. J. Groenendijk, M. Buscema, G. a. Steele, J. V. Alvarez, H. W. Zandbergen, J. J. Palacios and H. S. J. van der Zant, 2D Mater., 2014, 1, 025001.

30 J. D. Wood, S. A. Wells, D. Jariwala, K. Chen, E. Cho, V. K. Sangwan, X. Liu, L. J. Lauhon, T. J. Marks and M. C. Hersam, Nano Lett., 2014, 14, 6964-6970.

31 D. Yoon, H. Moon, Y.-W. Son, B. H. Park, J. B. Kim, Y. Lee and H. Cheong, Nano Lett., 2008, 8, 4270-4274. 